${ }^{\star}$ Corresponding author: s.weiler@ihfg.uni-stuttgart.de; Homepage: http://www.ihfg.uni-stuttgart.de

\title{
Post-selected indistinguishable single-photon emission from the Mollow triplet sidebands of a resonantly excited quantum dot
}

\author{
S. Weiler ${ }^{1} *$, D. Stojanovic ${ }^{1}$, S. M. Ulrich ${ }^{1}$, M. Jetter ${ }^{1}$, and P. Michler ${ }^{1}$ \\ ${ }^{1}$ Institut für Halbleiteroptik und Funktionelle Grenzflächen, \\ Universität Stuttgart, Allmandring 3, 70569 Stuttgart, Germany,
}

\begin{abstract}
Applying high power continuous-wave resonant s-shell excitation to a single self-assembled InGaAs quantum dot, we demonstrate the generation of post-selected single-indistinguishable photons from the Mollow triplet sidebands. A sophisticated spatial filtering technique based on a double Michelson interferometer enabled us to separate the spectrally close lying individual Mollow components and perform almost background free two-photon interference measurements. Showing high consistency with the results of an independent determination of the emission coherence of $\sim 250 \pm 30 \mathrm{ps}$, our analysis reveals a close to ideal visibility contrast of up to $97 \%$. Due to their easy spectral tunability and their distinct advantage of cascaded photon emission between the individual Mollow sidebands, they resemble a versatile tool for quantum information applications.
\end{abstract}


Investigations on the emission characteristics of single-photons sources such as trapped atoms [1], molecules [2] and quantum dots [3] are a rich field of science, especially in the focus of fundamental applications in quantum information technologies [4, 5]. The integratability of quantum dots (QDs) serving as deterministic single-photon sources, with the additionally ability to fulfill the often required precondition of emitting indistinguishable photons, make these solid state based "artificial atoms" attractive for intense research.

Several previous works on indistinguishable photon emission from single QDs relying on Hong-Ou-Mandel type two-photon interference at a beamsplitter [6] under non-resonant emitter state preparation have revealed visibilities up to $82 \%$ [7] 9 ].

These preceding studies have paved the way for several profound improvements for the application of QDs in quantum information technology, e.g. the proof of indistinguishable photon emission from spatially separate QDs [10, 11], as well as from photons emitted from mutually different sources. Here the bosonic nature in two-photon interference between QD emission and light created by parametric down-conversion has been successfully verified [12]. Another major achievement in terms of implementation has been the proof of indistinguishable photon emission from site-controlled single QDs [13].

Other ambitious developments focus on the creation of maximally indistinguishable photons. By employing strictly resonant emitter-state preparation, the visibility-limiting relaxation of electron-hole pairs from higher energetic QD states into its ground state is prohibited. Exploring the different regimes of resonance fluorescence, highly indistinguishable photons have been verified especially in the low excitation power limit so called Heitler regime [14-16]. Here the absorption and emission process becomes one coherent event and the scattered photons are free from any dephasing. In the regime close to emission saturation, non-classical two-photon interference has revealed indistinguishable photons both for continuous wave (cw) excitation [17], yielding a post-selected visibility contrast of $V_{H O M}=90 \%$, as well as for triggered resonant excitation with $\pi$-pulses [18] $\left(V_{H O M}=97 \%\right)$. The high-power dressed state regime, experimentally accessed with QDs by Muller et al. [19] for the first time, reveals the Mollow triplet as the characteristic hallmark of strongly driven resonance fluorescence. Single and cascaded photon emission between the Mollow sidebands has also been experimentally verified recently [20].

Inspired by these investigations, the aim of the current work is the verification and analysis of indistinguishable photons from the individual Mollow triplet sidebands with 
the distinct advantage of controlled spectral tunability via laser excitation power variation and/or resonance tuning.

In this letter we provide experimental evidence of post-selected indistinguishable photon emission from individual Mollow triplet sidebands of a cw resonantly excited single InGaAs QD for different pump powers, i.e. Rabi splittings. The coherence times of the sidebands extracted from independent Mollow triplet power series are, together with the relevant bunching and decay timescales derived from $g^{(2)}(\tau)$-type second-order auto-correlation measurements found to be in high consistency with the two-photon interference data.

The planar sample employed for the measurements in this work is grown by metalorganic vapor phase epitaxy. The self-assembled $\operatorname{In}(\mathrm{Ga})$ As QDs are embedded in a GaAs $\lambda$-cavity, sandwiched between 29 (4) periods of $\lambda / 4$-thick AlAs/GaAs layers as bottom (top) distributed Bragg reflectors. For our experimental investigations, the sample is kept in a Helium-flow cryostat providing a highly stable temperature of $T=5.0 \pm 0.5 \mathrm{~K}$. Suppression of parasitic laser stray-light is achieved by use of an orthogonal geometry between QD excitation and emission detection in combination with polarization suppression and spatial filtering via a pinhole in the detection path. The QDs are resonantly excited by a narrowband $(\approx 500 \mathrm{kHz})$ continuous-wave Ti:Sapphire ring laser. For high-resolution spectroscopy (HRPL) of micro-photoluminescence ( $\mu$-PL) we employ a scanning Fabry-Pérot interferometer with $\Delta E_{\mathrm{res}}^{\mathrm{HRPL}}<1 \mu \mathrm{eV}$ as described earlier [17, 20, 21].

A high degree of photon indistinguishability relies on the spectral purity of the detected photons. Therefore we utilize a double Michelson interferometer (motorized linear stages with a stage resolution of $20 \mathrm{~nm}$ ) in combination with a spectrometer (spectral resolution of $45 \mu \mathrm{eV}$ ) to selectively suppress and filter the distinct components of the Mollow triplet. As schematically depicted in Fig.1 (d) the first Michelson interferometer is set to the distinct path difference for which the two Mollow sidebands interfere constructively whereas the central Rayleigh peak is suppressed via destructive interference $: d=\lambda(\lambda+\Delta \lambda) /(2 \Delta \lambda)$. In a second similar filtering step either one of the Mollow sidebands is subsequently suppressed. A pre-condition of the applicability of this method is that the path difference between the two interferometer arms $l_{\text {path }}$ is well below the coherence length $l_{c o h}$ of the photons, which 
is readily fulfilled for our experimental conditions where $l_{\text {coh }}^{\min } \approx 71 \mathrm{~mm} \gg l_{\text {path }}^{\text {max }} \approx 14 \mathrm{~mm}$. Figure 1 (a)-(c) illustrates the functionality of the two-step double Michelson interferometer filtering process via high-resolution Mollow tiplet data.

Figure 2 shows a Mollow triplet power series of the QD under investigation. Because of the Fabry-Pérot-based technique in HRPL, all spectral features appear periodically with an offset equal to the free spectral range of the interferometer $\left(\Delta E_{F S R}=62.04 \mu \mathrm{eV}\right)$. With increasing excitation power, the evolution of two symmetrically spaced sidebands is clearly visible (black arrows) around the central line. To extract the evolution of coherence time $T_{2}$ of the Mollow sidebands a Lorentzian line shape has been fitted to the spectra. The Inset of Fig.2 shows the FWHM of the Mollow sidebands plotted versus the squared Rabi frequency $\Omega^{2}$, from which the expected linear increase due to excitation-induced dephasing is clearly observable [21, 22]. The herefrom calculated $T_{2}$-times are depicted in the same plot. For the experimental conditions of the two-photon interference measurements $\Omega=45.5 \mu \mathrm{eV}$ and $\Omega=53.8 \mu \mathrm{eV}, T_{2}$ is found to be $264 \pm 35 \mathrm{ps}$ and $237 \pm \mathrm{ps}$, respectively.

Figure 3 (I-III) presents the core of our investigations, i.e. Hong-Ou-Mandel type twophoton interference measurements on the individual Mollow triplet sidebands together with the auto-correlation measurements of the respective transition lines.

Figure 3 (I) shows the data set for a Rabi-splitting of $\Omega=45.5 \mu \mathrm{eV}$. The auto-correlation on the Mollow central peak is expected to reveal Poissonian statistics, due to the fact, that the emission of a Rayleigh photon does not modify the dressed-state population [23, 24]. As is clearly visible the Poissonian statistics is superimposed with a long-time bunching, as commonly observed due to "blinking" of the excitonic state between two or more neighboring competing states [25]. The phenomenon of "blinking" under pure-resonant excitation can be assigned to the presence of a competing QD exciton spin configuration, the nonradiative dark excitonic state [26]. The auto-correlation data has been fitted with a bidirectional exponential fit revealing a decay timescale of $\tau_{\text {bunch }}=9.51$ ns. Worth to note this power-dependent bunching is observed to superimpose all photon correlation measurements. High quality indistinguishability measurements crucially rely on the purity of single-photon emission, therefore auto-correlation measurements on the red Mollow sideband have been performed in advance, revealing $g^{(2)}(0)_{\text {deconv }}=0.12 \pm 0.03$ (considering the time-resolution 
of the setup of $400 \mathrm{ps}$ ). The correlation data unambiguously demonstrates single-photon emission with a fitted decay time constant of $\tau_{r}=540 \mathrm{ps}$. The finite background in the auto-correlation can be attributed to a small drift of the Michelson interferometer during the correlation measurement, allowing the spectrally close Mollow central peak to contribute to the detected signal as a non-vanishing uncorrelated background.

The Hong-Ou-Mandel type two-photon interference measurements shown in Fig. 3 (I) (c) and (d) have been performed under resonant cw excitation with an asymmetric fiberbased Mach-Zehnder interferometer (fixed interferometer delay $\Delta \tau=13 n s$ ). By a manually adjustable polarizer inserted into one interferometer arm the mutual photon polarization between the two inputs of arms entering the Hong-Ou-Mandel beamsplitter can be set orthogonal and therefore distinguishable where one expects $g^{(2)}(0)=0.5$. In parallel configuration the paths are indistinguishable and $g^{(2)}(0)$ should ideally become zero. The two-photon interference visibility calculated from the two different configurations is defined as $V_{H O M}(\tau)=\left[\left(g_{\perp}^{(2)}(\tau)-g_{\|}^{(2)}(\tau)\right)\right] / g_{\perp}^{(2)}(\tau)[17,[27]$.

The HOM data has then been modeled according to Ref. [11]. For this analysis the reflectivities $\mathrm{R}$ and transmittivities $\mathrm{T}$ of the beamsplitters have been measured to be $R=T=0.5 \pm 0.02$ and the wavepacket overlap at the Hong-Ou-Mandel beamsplitter as $V=0.98 \pm 0.02$. For fitting the data we use $T_{2}=300 \mathrm{ps}$ close to the extracted value of $T_{2}=264 \pm 35 \mathrm{ps}$, as well as $\tau_{\text {bunching }}$ and $\tau_{r}$ as derived from the auto-correlations discussed above. $g^{(2)}(0)$ for the parallel HOM correlation measurement is assumed to be slightly better than in (b) and (c), which was found to be related to a more stable positioning of the Michelson interferometers during the measurement time (less background from the Mollow central line). Considering the setup response we find $g_{\perp}^{(2)}(0)_{\text {deconv }}=0.61 \pm 0.04$ and $g_{\|}^{(2)}(0)_{\text {deconv. }}=0.04 \pm 0.01$, respectively. Therefore, a close to ideal post-selected visibility of $V_{H O M}=0.93 \pm 0.01$ (convoluted $V_{H O M}=0.47 \pm 0.08$ ) for the indistinguishability of the red Mollow sideband can be concluded.

Two-photon interference revealing indistinguishable photons has also been verified for a larger Rabi splitting of $\Omega=53.8 \mu \mathrm{eV}$ on the red and blue Mollow sideband, respectively. The measurements and evaluations are done similarly as described above. Fig. 3 (II) depicts the data for red Mollow sideband together with the auto-correlation on the central Mollow peak revealing a long-timescale bunching with $\tau_{\text {bunch }}=14.3 \mathrm{ps}$. The auto- 
correlation shows almost background-free single-photon emission with a deconvoluted value of $g^{(2)}(0)_{\text {deconv }}=0.08 \pm 0.02$. The timescale of the decay is found to be $\tau_{r}=570 \mathrm{ps}$ and the extracted coherence time derived from the HRPL power series of $T_{2}=237 \mathrm{ps}$ fits the data perfectly. Again a very high two-photon visibility of $V_{H O M}=0.97 \pm 0.02$ (convoluted $\left.V_{\text {HOM }}=0.43 \pm 0.07\right)$ has been extracted from the deconvoluted fits to the Hong-Ou-Mandel data.

Figure 3 (III) shows the correlation data of the blue sideband. $\tau_{r}$ and $T_{2}$ revealing full consistency with the measurements on the red Mollow sideband at the same Rabi splitting. The $g^{(2)}(0)_{\text {deconv }}$ is again found to be almost background free with a value of $0.03 \pm 0.01$. The Hong-Ou-Mandel visibility is calculated to be $V_{H O M}=0.92 \pm 0.02$ (convoluted $\left.V_{H O M}=0.39 \pm 0.08\right)$.

It is worth to mention that the decay time $\tau_{r}$ extracted from the auto-correlation measurements on the individual Mollow sidebands is found to be smaller than the theoretically predicted modified emission time of $\frac{\tau_{\text {rad }}}{\left(c^{4}+s^{4}\right)}=\tau_{m o d}$ for a resonantly exited quantum dot in the dressed-state regime. The two coefficients $c$ and $s$ are defined as $c=\sqrt{\frac{\Omega^{\prime}+\Delta}{2 \Omega^{\prime}}}$ $s=\sqrt{\frac{\Omega^{\prime}-\Delta}{2 \Omega^{\prime}}}$, where $\Omega^{\prime}=\sqrt{\Omega^{2}+\Delta^{2}}$ is the effective Rabi frequency and $\Delta$ the laser-detuning from resonance [23]. For out experimental conditions of strictly resonant excitation $\Delta=0$ we find $s=c=\frac{1}{\sqrt{2}}$. Independent time-resolved photoluminescence investigations under quasi-resonant emitter-state preparation have revealed a radiative lifetime of $\tau_{\text {rad }}=1008 \mathrm{ps}$, therefore the modified decay time for the system is calculated to be $\tau_{\text {mod }}=2016 \mathrm{ps}$. We emphasize that a similar discrepancy between experimentally extracted $\tau_{r}$ to theoretically calculated $\tau_{\text {mod }}$ has already been observed previously [20] and might be attributed to the distinctly different experimental conditions of cw resonant excitation to pulsed quasi-resonant emitter-state preparation. Nevertheless a profound explanation has to be left open for further in-depth analysis.

In conclusion, we have presented a detailed study of the indistinguishably of photons emitted from the Mollow triplet sidebands from a single semiconductor QD. Independently measured power series for emission coherence time extraction and auto-correlation measurements on the Mollow triplet central and sideband channels reveal full consistency with the cw indistinguishability measurements that exhibit high two-photon interference visibilities 
of up to $97 \%$.

We thank W.-M. Schulz for expert sample preparation, M. Heldmaier and A. Ulhaq for fruitful discussions. We acknowledge financial support of the DFG via the project MI500/231. S. Weiler appreciates funding from the Carl-Zeiss Stiftung.

[1] J. McKeever, A. Boca, A. D. Boozer, R. Miller, J. R. Buck, A. Kuzmich, and H. J. Kimble, Science 303, 1992 (2004).

[2] E. Lounis, and W. E. Moerner, Nature 407, 491-493 (2000).

[3] P. Michler, A. Kiraz, C. Becher, W. V. Schoenfeld, P. M. Petroff, Lidong Zhang, E. Hu, and A. Imamoglu, Science 209, 2282 (2000).

[4] J. L. O’Brien, A. Furusawa, and J. Vucković, Nat. Photon. 3, 687-695 (2009).

[5] H. J. Kimble, Nature 453, 1023-1030 (2008).

[6] C. K. Hong, Z. Y. Ou, and L. Mandel, Phys. Rev. Lett. 59, 2044-2046 (1987).

[7] S. Varoutsis, S. Laurent, P. Kramper, A. Lemaitre, I. Sagnes, I. Robert-Philip, and I. Abram, Phys. Rev. B 72, 041303(R) (2005).

[8] C. Santori, D. Fattal, J. Vučković, G. Solomon, and Y. Yamamoto, Nature 419, 594 (2002).

[9] S. Weiler, A. Ulhaq, S. M. Ulrich, S. Reitzenstein, A. Löffler, A. Forchel, and P. Michler, Phys. Status Solidi B 4, 867-871 (2011).

[10] E. B. Flagg, A. Muller, S. V. Polyakov, A. Ling, A. Migdall, and G. S. Solomon, Lett. Rev. Lett. 104, 137401 (2010).

[11] R. B. Patel, A. J. Bennett, I. Farrer, C. A. Nicoll, D. A. Ritchie, and A. J. Shields, Nature Photon. 4, 632-635 (2010).

[12] S. V. Polyakov, A. Muller, E. B. Flagg, A. Ling, N. Borjemscaia, E. Van Keuren, A. Migdall, and G. S. Solomon, Phys. Rev. Lett. 107, 157402 (2011).

[13] K. D. Jöns, P. Atkinson, M. Müller, M. Heldmaier, S. M. Ulrich, O. G. Schmidt, and P. Michler, Nano Lett. 13, 126-130 (2013).

[14] C. Matthiesen, M. Geller, C. H. H. Schulte, C. Le Gall, J. Hansom, Z. Li, M. Hugues, E. Clarke, and M. Atatüre, arXiv x, x (2012).

[15] C. Matthiesen, A. N. Vamivakas, and M. Atatüre, Phys. Rev. Lett. 108, 093602 (2012). 
[16] H. S. Nguyen, G. Sallen, C. Voisin, P. Roussignol, C. Diederichs, and G. Cassabois, Appl. Phy. Lett. 99, 261904 (2011).

[17] S. Ates, S. M. Ulrich, S. Reitzenstein, A. Löffler, A. Forchel, and P. Michler, Phys. Rev. Lett. 103, $167402(2009)$.

[18] Y.-M. He, Y. He, Y.-J. Wei, D. Wu, M. Atatüre, C. Schneider, S. Höffling, M. Kamp, C.-Y. Lu and J.-W. Pan, Nature Nanotech. x, x (2013).

[19] A. Muller, E. B. Flagg, P. Bianucci, X. Y. Wang, D. G. Deppe, W. Ma, J. Zhang, G. J. Salamo, M. Xiao, and C. K. Shih, Lett. Rev. Lett. 99, 187402 (2007).

[20] A. Ulhaq, S. Weiler, S. M. Ulrich, R. Rossbach, M. Jetter, and P. Michler, Nature Photonics 6, 238-242 (2012).

[21] S. M. Ulrich, S. Ates, S. Reitzenstein, A. Löffler, A. Forchel, and P. Michler, Phys. Rev. Lett. 106, $247402(2011)$.

[22] C. Roy, and S. Hughes, Phys. Rev. Lett. 106, 247403 (2011).

[23] C. A. Schrama, G. Nienhuis, H. A. Dijkerman, C. Steijsiger, and H. G. M. Heideman, Phys. Rev. A 45, 8045-8055 (1992).

[24] G. Nienhuis, Phys. Rev. A 47, 510-518 (1993).

[25] C. Santori, M. Pelton, G. Solomon, Y. Dale, and Y. Yamamoto, Phys. Rev. Lett. 86, 1502-1505 (2001).

[26] M. Bayer, G. Ortner, O. Stern, A. Kuther, A. A. Gorbunov, A. Forchel, P. Hawrylak, S. Fafard, K. Hinzer, T. L. Reinecke, S. N. Walck, J. P. Reithmaier, F. Klopf, and F. Schäfer, Phys. Rev. B 65, 195315 (2002).

[27] R. B. Patel, A. J. Bennett, K. Cooper, P. Atkinson, C. A. Nicoll, D. A. Ritchie, and A. J. Shields, Lett. Rev. Lett. 100, 207405 (2008). 


\section{Figures}

Figure 1, S. Weiler
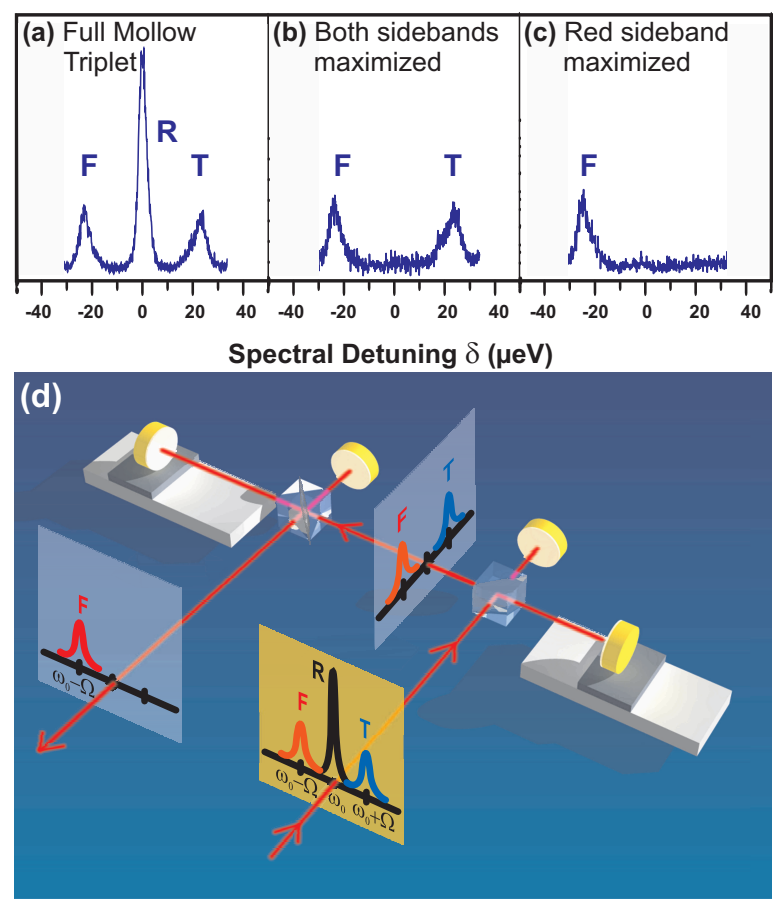
Figure 2, S. Weiler

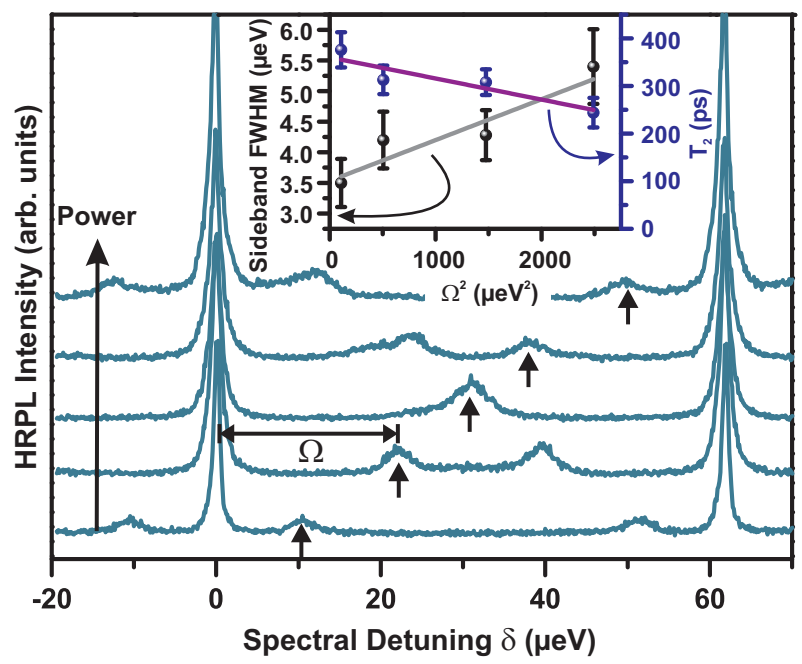


Figure 3, S. Weiler
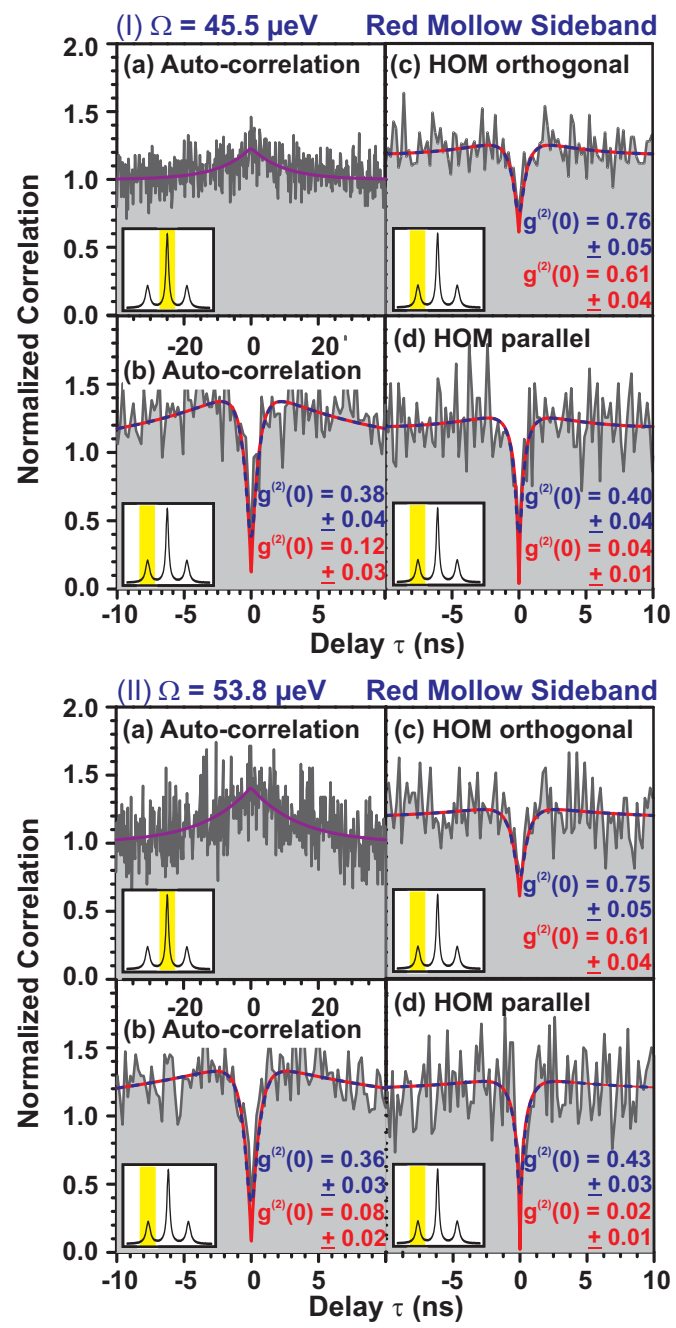

(III) $\Omega=53.8 \mu \mathrm{eV}$ Blue Mollow Sideband

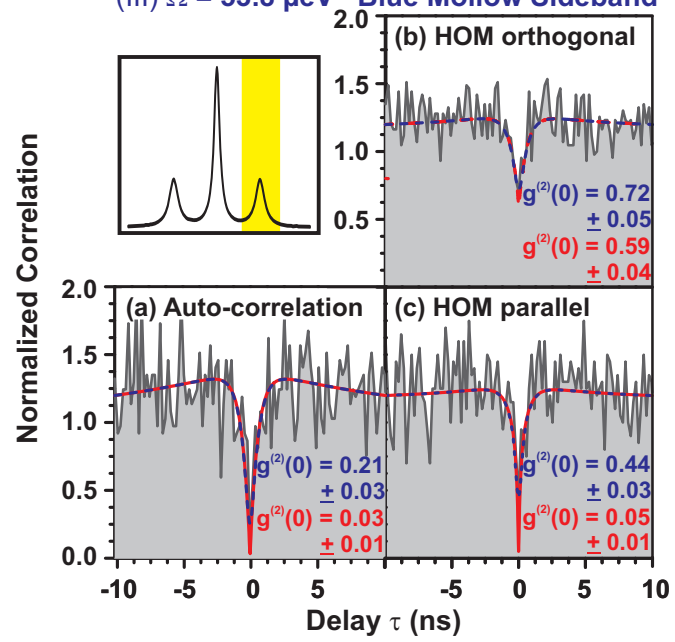




\section{Figure Captions}

\section{FIG.1}

Double Michelson Filtering Technique: High-resolution spectrum of (a) The full Mollow triplet without Michelson filtering (b) The Mollow triplet sidebands (suppressed Rayleigh line after first filtering step) (c) Only the red Mollow sideband (after second filtering step). (d) Illustration of the double Michelson interferometer setup used to spatially separate one Mollow component from the other two.

\section{FIG.2}

Coherence Time Extraction: Power dependent Mollow triplet spectra. The belonging sidebands are marked with black arrows, the additional peaks reflect the different orders of interference of the scanning Fabry-Pérot interferometer. Inset: FWHM of the Mollow sidebands, extracted from Lorentzian fits to the HRPL data and the herefrom calculated coherence time $T_{2}$ versus $\Omega^{2}$.

\section{FIG.3}

Correlation Measurements: Blue dashed (red straight) line convoluted (deconvoluted) fits to the $g^{(2)}(\tau)$ data.

(I) Data set for the two-photon interference measurements of the red Mollow sideband for $\Omega=45.5 \mu \mathrm{eV}$. (a) Auto-correlation of the central Mollow peak revealing long-term bunching with a timescale of $\tau_{\text {bunch }}=9.51$ ns. (b)-(d) Auto-correlation, perpendicular and parallel Hong-Ou-Mandel measurements on the photon emission of the red Mollow sideband. The fits reveal a modified emission time of $\tau_{r}=540 \mathrm{ps}$ and $T_{2}=300 \mathrm{ps}$ close to the independently derived coherence time of 264 ps from the HRPL spectra. The orthogonal HOM data is in full consistency with these values, for the parallel case $g^{(2)}(0)$ has been assumed to be slightly better than for the other measurements. The deconvoluted values reveal a high degree of two-photon visibility $V_{H O M}=0.93 \pm 0.01$ (convoluted $V_{H O M}=0.47 \pm 0.08$ ).

(II) Similar data set than in (I) but at for $\Omega=53.8 \mu \mathrm{eV}$. The values for fitting all correlation measurements are found to be $\tau_{\text {bunch }}=14.3 \mathrm{ps}, T_{2}=237 \mathrm{ps}, \tau_{r}=570 \mathrm{ps}$, exhibiting a visibility of $V_{H O M}=0.97 \pm 0.02$ (convoluted $V_{H O M}=0.43 \pm 0.07$ ).

(III) Auto- and HOM correlations for the blue Mollow sideband at $\Omega=53.8 \mu \mathrm{eV}$. The fit- 
ting values $\tau_{r}=570 \mathrm{ps}$ and $T_{2}=237 \mathrm{ps}$ are in accordance with all correlation measurements. Here the evaluations reveals $V_{H O M}=0.92 \pm 0.02$ (convoluted $V_{H O M}=0.39 \pm 0.08$ ). 